\title{
Management of the Axilla and the Breast After Neoadjuvant Chemotherapy in Patients with Breast Cancer: A Systematic Review
}

\author{
Bulent Citgez, (1) Banu Yigit, (1) Sitki Gurkan Yetkin
}

Department of General Surgery, University of Health Sciences Turkey, Sisli Hamidiye Etfal Teaching and Research Hospital, Istanbul Turkey

\begin{abstract}
Breast cancer is the most common cancer in women worldwide. Breast cancer is traditionally treated with surgery, plus adjuvant systemic therapy and radiotherapy as required. Neoadjuvant chemotherapy (NACT) for the treatment of breast cancer is used for locally advanced operable breast cancer to reduce the tumor size, to perform breast conserving surgery, and to perform a limited axillary approach. Adjuvant chemotherapy for the treatment of inflammatory breast cancer and even in inoperable breast cancer is used to increase overall survival time and to delay disease progression while relieving symptoms. NACT for breast cancer is a new strategy that was introduced toward the end of the $20^{\text {th }}$ century and is increasingly used in the treatment of breast cancer. At present, NACT is increasingly being used to reduce the need for axillary dissection and to convert patients with large tumors to candidates for breast conservation therapy in both locally advanced and operable breast cancers. Breast conserving procedures are currently more preferred by surgeons and axillary dissection is being replaced by sentinel lymph node biopsy after chemotherapy. One of the targets of neoadjuvant systemic therapy is to try to perform a less aggressive surgery by breast conservation, mainly for cosmetic reasons and avoiding axillary dissection mainly for arm mobility, pain, and lymphedema risk. The other target of neoadjuvant systemic therapy is to see the response of the tumor to chemotherapy and determine the treatment accordingly. Neoadjuvant systemic therapy increases the rate of complete pathological response by clearing the breast and axilla from tumor cells before surgery. In this review, we examine the key points of using the NACT in breast cancer, considering radiological imaging methods, surgical management, and reconstruction after NACT.
\end{abstract}

Keywords: Axillary dissection; breast cancer; neoadjuvant chemotherapy; pathological complete response; radiotherapy; surgery; reconstruction.

Please cite this article as "Citgez B, Yigit B, Yetkin SG. Management of the Axilla and the Breast After Neoadjuvant Chemotherapy in Patients with Breast Cancer: A Systematic Review. Med Bull Sisli Etfal Hosp 2021;55(2):156-161".

$\mathrm{O}$ ver the decades, breast cancer surgery evolved from radical mastectomies to more conservative techniques to breast conserving surgery (BCS) and axillary sentinel lymph node (SLN) biopsy. For locally advanced and some early stage breast cancer patients, neoadjuvant chemotherapy (NACT) is widely accepted. Surgery following NACT is a standard treatment approach. NACT increases resectability of locally advanced breast cancer, rate of BCS, and cosmetic outcomes; decreases the morbidity and extent of axillary surgery; improves quality of life; and improves prognosis in selected disease subtypes (especially triple-negative [TN] breast cancer subtype) with pathological complete re-

Address for correspondence: Bulent Citgez, MD. Saglik Bilimleri Universitesi, Sisli Hamidiye Etfal Egitim ve Arastirma Hastanesi,

Genel Cerrahi Anabilim Klinigi, Istanbul, Turkey

Phone: +90 5052376847 E-mail: bcitgez@yahoo.com

Submitted Date: November 12, 2020 Accepted Date: March 25, 2021 Available Online Date: July 02, 2021

${ }^{\circ}$ Copyright 2021 by The Medical Bulletin of Sisli Etfal Hospital - Available online at www.sislietfaltip.org

OPEN ACCESS This is an open access article under the CC BY-NC license (http://creativecommons.org/licenses/by-nc/4.0/). 
sponse compared to partial responders. Another advantage of NACT is to see the response of the tumor to chemotherapy and determine the treatment accordingly. ${ }^{[1,2]}$ Complete response includes patients without residual invasive carcinoma in the breast and lymph nodes and partial response is defined as a decrease or no increase in either or both tumor size $(\mathrm{T})$ or lymph node status $(\mathrm{N})$ stage compared to the pretreatment $\mathrm{T}$ or N. Grade, tumor biology, and genomic testing results are now considered in conjunction with tumor size and lymph node involvement to determine breast cancer stage. Therefore, the ratio of partial and complete response rates according to breast cancer stages varies depending on all these variables. ${ }^{[3]}$ Reconstruction remains an option for patients managed with NACT; discussion must be multidisciplinary and initiate before treatment. Complicating the decision regarding the technique and timing of breast reconstruction is the potential need for post-mastectomy radiotherapy (RT). ${ }^{[4]}$ Breast cancer treatment has evolved into a multidisciplinary approach, requiring a team that includes surgeons, clinical and medical oncologists, radiologists, and pathologists in patient care.

\section{Clinical Impact of Residual Disease After NACT}

NACT has become the standard of care for the treatment of patients with clinically node-positive $(\mathrm{cN}+)$ breast cancer and can eradicate axillary metastasis in $40-75 \%$ of patients, depending on tumor subtype. The pathologic complete response $(p C R)$ after NACT is $10-15 \%$ with anthracyclines, $25-30 \%$ with the addition of taxanes, $50 \%$ with the addition of trastuzumab, and $60 \%$ with the addition of pertuzumab. Survival is worse, especially in patients with TN and HER2 overexpressing (HER2+) tumors who do not have a pCR. ${ }^{[5]}$

IMpassion130 study evaluated nab-paclitaxel chemotherapy plus atezolizumab versus placebo in TN advanced stage breast cancer. This study showed persistent benefit of atezolizumab plus nab-paclitaxel only for PD-L1-positive tumors. Keynote 522 study showed that $\mathrm{PCR}$, assessed in the first 602 patients, significantly increased from $51.2 \%$ in the placebo group to $64.8 \%$ in the pembrolizumab group. Since TN breast cancer is aggressive and recurrences often occur early on, addition of pembrolizumab to NACT prevents breast cancer recurrence in TN breast cancer group. Recurrence risk score is lower and pCR rates $(<10 \%)$ are lower in estrogen receptor (ER)/progesterone receptor (PR)-positive patients receiving NACT because of the biology of the disease. Changes in the Ki- 67 level in the early period also indicate this. ${ }^{[6,7]}$

Residual disease is measured by residual cancer burden $(\mathrm{RCB})$, neoadjuvant response index (NRI), and Neo-Bioscore. ${ }^{[8]} \mathrm{NRI}$ is a value calculated based on the response to neo- adjuvant therapy of the breast and lymph nodes, and is correlated with recurrence-free survival in the presence of TN subtype. Values between 0 and 1 are given; 0 - no answer and 1 - considered as pCR. Neo-Bioscore; clinical stage, post-neoadjuvant pathological stage, ER and HER2 status, and nuclear grad are taken into account and seven response categories are formed. In RCB, the diameter of the residual disease, the percentage of viable tumor cells, and the largest affected $L N$ diameter are measured. Score between 0 and 3 is given: RCB $0, p C R$ in axilla and breast; $R C B$ 1, minimal residual disease; RCB2, moderate residual disease; and RCB3 represents extensive residual disease.

The CREATE- $X$ trial investigates the role of adjuvant capecitabine in patients with residual tumors in the HER2group after NACT. For those with TN breast cancer subtype, two randomized trials, EA1131 and S1418, are ongoing. The aim of the EA1131 study was to compare the good and bad effects of capecitabine or platinum-based chemotherapy (cisplatin or carboplatin) after surgery for TN groups with $>1 \mathrm{~cm}$ residual disease who received neoadjuvant therapy. In the $\mathrm{S} 1418$ study, patients with $>1 \mathrm{~cm}$ residual disease, pathological node positive (ypN+), and non-metastatic TN patients after NACT were randomized as adjuvant pembrolizumab therapy or placebo. In the KATHERINE trial, among the patients with HER2 + CT1-4/N0-3/M0 with residual disease after completion of neoadjuvant therapy ( 6 cycles of chemotherapy $[\mathrm{CT}]$ and should have received a minimum of 9 weeks), the rate of invasive breast cancer recurrence was compared among patients who received trastuzumab only and T-DM1 as adjuvant therapy. Recurrence rate in the patients receiving T-DM1 was found $50 \%$ lower and the survival was significantly higher. The PENELOPE study aims to demonstrate that palbociclib provides superior invasive disease-free survival (iDFS) in women with locally advanced breast cancer with a higher risk of recurrence after hormone receptor (HR)-positive and HER2-positive patients have less $\mathrm{pCR}$ to chemotherapy with neoadjuvant taxane against the background of standard anti-hormonal therapy ${ }^{\left[{ }^{[9]}\right.}$

As a result, TN and HER2+ breast cancer patients with T2 or larger tumors or affected lymph nodes are candidates for neoadjuvant therapy. In HR+ group, $\mathrm{pCR}$ is lower but in this group, the biology of the disease is better. Additional adjuvant therapy is needed in patients without $\mathrm{pCR}$. Studies on the use of capecitabine in TN and high-risk luminal, HER2+ group; TDM1 in HER2+ groups are ongoing.

\section{Radiological Imaging Studies After NACT}

The primary purpose of radiological imaging studies after NACT is to predict residual tumor size. Magnetic resonance imaging (MRI) is a reliable radiological imaging for 
assessing tumor response after NACT because it allows us to distinguish between non-vascularized therapy-induced fibrosis and residual vital tumor. When evaluating tumor regression with $\mathrm{MRl}$; if there is a mass lesion, tumor size should be measured as the longest diameter. For non-mass lesions, the entire contrast enhancement is evaluated as a single lesion. Tumor regression can be assessed on MRI by regression shrinkage patterns. The shrinkage pattern was seen in three types: Concentric, nodular, and mixed (both concentric and nodular). Evaluation of the tumor response to NACT by MRI influenced by the molecular breast cancer subtypes. Evaluation is more accurate in TN and HER2+ tumors because the tumor is more likely to appear as a unifocal mass and shrinks in a concentric type. Luminal subtypes of breast cancer are more likely to appear as a diffuse lesion and regression in the form of fragmentation. Therefore, we have difficulty in evaluating regression. The NACT type may also affect MRI accuracy. Taxanes have more antiangiogenic activity than drugs with anthracycline, may decrease contrast enhancement, which would produce an underestimation. ${ }^{[10]}$ If there is reactive inflammation and fibrosis around the tumor bed, MRI may overestimate residual disease. Residual ductal carcinoma in situ (DCIS) or small residual lesion after NACT may lead to misinterpretation. Residual DCIS may lead to false positive diagnosis, while small residual lesion may lead to false-negative diagnosis. ${ }^{[11,12]}$ Radiologic complete response ( $r C R)$ with MRI is not always $p C R$. In a retrospective study by De Los Santos et al., ${ }^{[13]}$ overall accuracy of MRI for predicting pCR was found to be $74 \%$. MRI correlated well with evaluating response of TN and HER2+ tumors but not for luminal subtypes, this possibly influenced by differences in $\mathrm{pCR}$ rates between groups. ${ }^{[14]}$

Although response to NACT is commonly evaluated by ultrasound (US), mammography, and MRI, studies on the accuracy of positron emission tomography/computed tomography (PET/CT) for the evaluation of response to NACT have been encouraging. MRI had a higher specificity but PET/CT had a higher sensitivity for predicting the pathologic response after NACT in patients with breast cancer. ${ }^{[15]}$ It appears that significant reduction in cellular glycolysis induced by effective cytotoxic drugs and a bigger decrease in SUVmax following the early cycles of NACT are indicative of better histopathological status after the final course of NACT. ${ }^{[16]}$

Studies are still ongoing for non-surgical follow-up in patients with $\mathrm{pCR}$ after NACT and to identify the patients with pCR by performing image-guided biopsy of the tumor bed, preferably with vacuum-assisted core biopsy, in patients with rCR. However, it has been known so far that biopsy methods are insufficient to exclude residual disease and the patients who achieve $p C R$ still require surgical excision of the tumor bed. Even if those patients with positive HR status and low-grade tumors achieve rCR, they are associated with residual disease at surgery. ${ }^{[17,18]}$

Axillary US (AUS) can be used to restage the axilla or to assess the axilla to evaluate the axillary nodes for evidence of metastatic disease with good accuracy after NACT in women with node positive at diagnosis. Morphological criteria used in the evaluation of axillary lymph nodes with US were based on the cortex and hilum of the lymph node. A lymph node is considered abnormal if the cortex is thickened $(>3$ $\mathrm{mm}$ thick), the fatty hilum is obliterated by dense capsule, and the lymph node is round in shape. US, in particularly, allows us to assess level 1 and 2 axillary lymph nodes better. MRI has a slightly lower sensitivity with $64 \%$ in showing residual metastasis, but level 3 axillary lymph nodes and internal mammary lymph nodes are assessed better. The ACOSOG Z1071 Trial reported that the false negativity rate (FNR) for SLN surgery after NACT in patients with cN+ disease and two or more SLNs removed at the time of surgery were $12.6 \%$ at tolerable high level. Studies have been carried out in the form of whether the AUS can replace SLN biopsy (SLNB) or the addition of AUS can decrease the FNR of SLNB. ${ }^{[19]}$ Sometimes, the identified lymph node may not be SLN. It is not clear what the cortex thickness cutoff value should be, sometimes, it is considered $>2.5 \mathrm{~mm}$ or $>3 \mathrm{~mm}$. If lymph nodes are deeply located, isolated tumor cell or micrometastases may not be displayed sonographically. It has been seen that AUS has many limitations and cannot replace SLNB but lowers the FNR after NACT. ${ }^{[20]}$

Consequently, AUS should be performed before NACT, biopsy should be performed on the suspicious lymph node in terms of metastasis and clips should be placed in nodes with biopsy-confirmed metastasis. Reevaluation of the axilla after NACT should be made with AUS again, which will guide surgical decision-making and reduces FNR of SLNB after NACT. When no residual disease identified in the axillary lymph nodes after radiological imaging studies and retrieval of $\geq 3$ sentinel nodes at SLNB, there is no need for axillary dissection and it avoids the complications associated with axillary lymph node dissection (ALND).

\section{Surgery After NACT}

The important thing here is whether to conserve the breast or to prevent axillary dissection. Surgical planning and drug regimens are selected by assessing tumor response by imaging studies.

\section{Axilla}

At this stage, it is important to determine whether the patient has lymph node involvement before neoadjuvant therapy. SLNB is possible after axillary downstaging. It 
seems rational to assume that aggressive surgical treatment of the axilla with an ALND might be omitted in patients in whom an axillary $\mathrm{PCR}$ is achieved since the axilla has already been cleared of tumor deposits by systemic treatment. It has been observed that the targeted axillary dissection (TAD), which includes SLNB and selective localization and removal of clipped nodes using iodine-125 seed localization, increases the accuracy of axillary staging and improves the FNR compared with SLNB alone. In a study by Caudle et al., ${ }^{[21]} 191$ patients with nodal metastases who completed NACT followed by SLNB and ALND were enrolled to determine the FNR of SLNB. Clips were placed in nodes with biopsy-confirmed metastasis before initiating NACT. The FNR for patients undergoing SLNB and ALND was $10.1 \%$, for the evaluation of the clipped node alone was $4.2 \%$, and for patients who received TAD followed by ALND was $2.0 \%$. It was observed that the clipped node was not retrieved as an SLN in 23\% and the use of dual tracers lowered the FNR.

Neoadjuvant endocrine therapy becomes an attractive option for selected patients with hormonal receptor positive locally advanced breast cancer. Neoadjuvant endocrine therapy never increases the axillary dissection rate in clinically node-negative ( $\mathrm{cN}-$ ) patients. Although survival is higher in node-negative patients than positive patients, there is no difference in survival between those who were initially operated and those who were operated after neoadjuvant endocrine therapy. ${ }^{[2]}$

\section{Breast}

NACT is a valuable treatment method for downstaging of tumor size without increasing the risk of locoregional recurrence, thus allowing BCS in many patients who would have previously supposed to undergo mastectomy procedure. It is important to see the effectiveness of CT and obtain prognostic information. $\mathrm{pCR}$ is defined as no residual or in situ disease determined in the breast. Patients, who have $\mathrm{pCR}$ or minimal residual burden following NACT, have longer disease-free survival (DFS) and overall survival and low risk of recurrence. Therefore, the tumor bed should be marked radiologically before NACT. In the future, when the radiological predictability of the $\mathrm{PCR}$ increases, there would be a possibility of omitting surgery and the patient could be safely treated with RT alone. ${ }^{[23,24]}$

Clinical response (cN+ vs. - , HER2+ vs. -, TN, luminal A vs. B, and Grade 3 vs. Grade 1) should be evaluated on a case-bycase basis. According to the tumor receptor, the response type that can be received in the breast to NACT is different, so the surgical limit should be considered in the residual tumor according to the response type. TN and HER2+ tumors shrink concentrically to form a single foci of residual tumor cells while ER/PR (+) and HER2- leave scattered tumor cells in patchy patterns. The rate of $\mathrm{pCR}$ is higher in HER2+ and TN breast cancers, and less frequent in HR-positive breast cancers. Although $\mathrm{pCR}$ rates differ, there is no indication for wider surgical margins because of the molecular subtype, multimodality treatment is important. Resection of the clipped area in cases of $\mathrm{pCR}$ is recommended. It is accepted that the approach of no tumor cells in the resection margin is sufficient. ${ }^{[25]}$ Whether there is a residual tumor which is important for post-operative adjuvant therapy because adjuvant therapy increases survival. If we do not notice the residual tumor, the patient will lose the chance of adjuvant treatment. ${ }^{[26,27]}$

Consequently, many randomized studies are ongoing to avoid surgery or aim to after a pCR, but surgery is still mandatory. Less radical surgery of the breast and axillary lymph nodes is possible.

\section{Reconstruction After NACT}

There has been discussion about the timing and usage of adjuvant radiotherapy, hormonal therapy, and chemotherapy with respect to surgical interventions. Implant-based breast reconstructions are mostly preferred for reconstruction after NACT. There are two common methods of implantbased breast reconstruction, one-stage reconstruction and two-stage reconstruction. One-stage implant-based breast reconstruction is a reconstructive method performed at the time of mastectomy without the need for tissue expander. Two-stage implant-based breast reconstruction with initial insertion of a tissue expander followed by changeover to an implant after a period of inflation can be performed by months or years after initial treatment. In a study by Teotia et al., ${ }^{[28]} 128$ patients were retrospectively evaluated. It was shown that the time elapsed after the last cycle of NACT was not significant in terms of post-operative complications. High body mass index and usage of tissue expander were determined as factors increasing complications. In a review by Riba et al., ${ }^{[29]}$ the results of different studies were evaluated retrospectively. In neoadjuvant breast cancer groups, use of an autologous flap in previously irradiated breasts appears to reduce the incidence of implant-related complications; studies have shown that rates of implantation loss can reach up to $25 \%$ in implant-based breast reconstruction group.

Especially in the neoadjuvant group, we see that repair with one-stage implant-based breast reconstruction compared to two-stage implant-based reconstruction reduces implant losses and does not delay the adjuvant treatment process. A meta-analysis performed by Ricci et al. ${ }^{[30]}$ showed that relative to permanent implants, post-mastec- 
tomy RT applied tissue expanders resulted in higher rates of reconstructive failure (20\% with expander + RT vs. $13,4 \%$ with implant + RT) and lower rates of capsule contracture (24,5\% with expander + RT vs. $49.4 \%$ with implant + RT). Compared to tissue expanders placed below the pectoralis muscle, tissue expanders placed above the pre-pectoral area accelerate the post-operative expansion process, provide faster recovery and better vascularization with high performance soft-tissue support, and carry low risk of seroma so that the patients undergo post-operative RT treatment faster. ${ }^{[31]}$

Consequently, RT becomes important in young women with invasive breast cancer, as the risk of local recurrence is higher than in older women, reconstructed breast mounds that underwent RT had significantly higher associations with implant or tissue expander exposure. If complications develop after tissue expander placement with a history of radiation therapy, early conversion to autologous techniques should be performed.

\section{Conclusion}

NACT in breast cancer eradicates micrometastatic disease, decreases extent of surgery, provides prognostic information, and identifies candidates for additional treatment approaches.

\section{Disclosures}

Peer-review: Externally peer-reviewed.

Conflict of Interest: None declared.

Financial Disclosure: The authors declare that this study received no financial support.

Authorship Contributions: Concept - B.C., B.Y.; Design - S.G.Y; Supervision - B.C., S.G.Y.; Fundings - B.C.; Materials - B.C.; Data collection \&/or processing - B.Y.; Analysis and/or interpretation - S.G.Y.; Literature search - B.C, B.Y.; Writing - B.C, B.Y.; Critical review - S.G.Y.

\section{References}

1. Volders JH, Negenborn VL, Spronk PE, Krekel NMA, Schoonmade LJ, Meijer S, et al. Breast-conserving surgery following neoadjuvant therapy-a systematic review on surgical outcomes. Breast Cancer Res Treat 2018;168:1-12. [CrossRef]

2. Petruolo O, Sevilimedu V, Montagna G, Le T, Morrow M, Barrio AV. How often does modern neoadjuvant chemotherapy downstage patients to breast-conserving surgery? Ann Surg Oncol 2021;28:287-94. [CrossRef]

3. Giuliano AE, Connolly JL, Edge SB, Mittendorf EA, Rugo HS, Solin $\mathrm{LJ}$, et al. Breast Cancer-Major changes in the American Joint Committee on Cancer eighth edition cancer staging manual. CA Cancer J Clin 2017;67:290-303. [CrossRef]

4. Ho AY, Hu ZI, Mehrara BJ, Wilkins EG. Radiotherapy in the setting of breast reconstruction: types, techniques, and timing. Lancet Oncol 2017;18:e742-53. [CrossRef]

5. Cortazar P, Zhang L, Untch M, Mehta K, Costantino JP, Wolmark $\mathrm{N}$, et al. Pathological complete response and long-term clinical benefit in breast cancer: the CTNeoBC pooled analysis. Lancet 2014;384:164-72. [CrossRef]

6. Schmid P, Adams S, Rugo HS, Schneeweiss A, Barrios CH, Iwata $\mathrm{H}$, et al; IMpassion130 Trial Investigators. Atezolizumab and nab-paclitaxel in advanced triple-negative breast cancer. $N$ Engl J Med 2018;379:2108-21. [CrossRef]

7. Schmid P, Cortes J, Pusztai L, McArthur H, Kümmel S, Bergh J, et al; KEYNOTE-522 Investigators. Pembrolizumab for Early Triple-Negative Breast Cancer. N Engl J Med 2020;382:810-21. [CrossRef]

8. Steenbruggen TG, van Seijen M, Janssen LM, van Ramshorst MS, van Werkhoven E, Vrancken Peeters MTDF, et al. Prognostic value of residual disease after neoadjuvant therapy in her2-positive breast cancer evaluated by residual cancer burden, neoadjuvant response index, and neo-bioscore. Clin Cancer Res 2019;25:498592. [CrossRef]

9. Von Minckwitz G, Bear H, Bonnefoi H, Colleoni M, Gelmon K, Gnant $M$ et al. Abstract OT2-6-11: PENELOPE: Phase III study evaluating palbociclib (PD-0332991), a cyclin-dependent kinase (CDK) 4/6 inhibitor in patients with hormone-receptor-positive, HER2-normal primary breast cancer with high relapse risk after neoadjuvant chemotherapy (GBG-78/BIG1-13). Cancer Res 2013;73:Abstract nr OT2-6-11. [CrossRef]

10. Ko ES, Han BK, Kim RB, Ko EY, Shin JH, Hahn SY, et al. Analysis of factors that influence the accuracy of magnetic resonance imaging for predicting response after neoadjuvant chemotherapy in locally advanced breast cancer. Ann Surg Oncol 2013;20:2562-8.

11. Denis F, Desbiez-Bourcier AV, Chapiron C, Arbion F, Body G, Brunereau $L$. Contrast enhanced magnetic resonance imaging underestimates residual disease following neoadjuvant docetaxel based chemotherapy for breast cancer. Eur J Surg Oncol 2004;30:106976. [CrossRef]

12. Chen JH, Feig B, Agrawal G, Yu H, Carpenter PM, Mehta RS, et al. $\mathrm{MRI}$ evaluation of pathologically complete response and residual tumors in breast cancer after neoadjuvant chemotherapy. Cancer 2008;112:17-26. [CrossRef]

13. De Los Santos JF, Cantor A, Amos KD, Forero A, Golshan M, Horton $\mathrm{JK}$, et al. Magnetic resonance imaging as a predictor of pathologic response in patients treated with neoadjuvant systemic treatment for operable breast cancer. Translational Breast Cancer Research Consortium trial 017. Cancer 2013;119:1776-83. [CrossRef]

14. Namura M, Tsunoda $H$, Kobayashi D, Enokido K, Yoshida A, Watanabe $T$, et al. The loss of lymph node metastases after neoadjuvant chemotherapy in patients with cytology-proven axillary node-positive primary breast cancer. Clin Breast Cancer 2019;19:278-85. [CrossRef]

15. Liu Q, Wang C, Li P, Liu J, Huang G, Song S. The role of (18)F-FDG $\mathrm{PET} / \mathrm{CT}$ and $\mathrm{MRI}$ in assessing pathological complete respon- 
se to neoadjuvant chemotherapy in patients with breast cancer: a systematic review and meta-analysis. Biomed Res Int 2016;2016:3746232. [CrossRef]

16. Paydary K, Seraj SM, Zadeh MZ, Emamzadehfard S, Shamchi SP, Gholami $S$, et al. The evolving role of FDG-PET/CT in the diagnosis, staging, and treatment of breast cancer. Mol Imaging Biol 2019;21:1-10. [CrossRef]

17. Rauch GM, Kuerer HM, Adrada B, Santiago L, Moseley T, Candelaria RP, et al. Biopsy Feasibility trial for breast cancer pathologic complete response detection after neoadjuvant chemotherapy: imaging assessment and correlation endpoints. Ann Surg Oncol 2018;25:1953-60. [CrossRef]

18. Kuerer HM, Rauch GM, Krishnamurthy S, Adrada BE, Caudle AS, DeSnyder SM, et al. A clinical feasibility trial for identification of exceptional responders in whom breast cancer surgery can be eliminated following neoadjuvant systemic therapy. Ann Surg 2018;267:946-51. [CrossRef]

19. Boughey JC, Suman VJ, Mittendorf EA, Ahrendt GM, Wilke LG, Taback $B$, et al; Alliance for Clinical Trials in Oncology. Sentinel lymph node surgery after neoadjuvant chemotherapy in patients with node-positive breast cancer: the ACOSOG Z1071 (Alliance) clinical trial. JAMA 2013;310:1455-61. [CrossRef]

20. Boughey JC, Ballman KV, Hunt KK, McCall LM, Mittendorf EA, Ahrendt GM, et al. Axillary ultrasound after neoadjuvant chemotherapy and its impact on sentinel lymph node surgery: results from the American College of Surgeons Oncology Group Z1071 Trial (Alliance). J Clin Oncol 2015;33:3386-93. [CrossRef]

21. Caudle AS, Yang WT, Krishnamurthy S, Mittendorf EA, Black DM, Gilcrease $M Z$, et al. Improved axillary evaluation following neoadjuvant therapy for patients with node-positive breast cancer using selective evaluation of clipped nodes: implementation of targeted axillary dissection. J Clin Oncol 2016;34:1072-8. [CrossRef]

22. Kim HJ, Noh WC, Lee ES, Jung YS, Kim LS, Han W, et al. Efficacy of neoadjuvant endocrine therapy compared with neoadjuvant chemotherapy in pre-menopausal patients with oestrogen receptor-positive and HER2-negative, lymph node-positive breast cancer. Breast Cancer Res 2020;22:54. [CrossRef]
23. Mamtani A, Barrio AV, King TA, Van Zee KJ, Plitas G, Pilewskie M, et al. How often does neoadjuvant chemotherapy avoid axillary dissection in patients with histologically confirmed nodal metastases? Results of a prospective study. Ann Surg Oncol 2016;23:3467-74. [CrossRef]

24. Pilewskie M, Zabor EC, Mamtani A, Barrio AV, Stempel M, Morrow $M$. The optimal treatment plan to avoid axillary lymph node dissection in early-stage breast cancer patients differs by surgical strategy and tumor subtype. Ann Surg Oncol 2017;24:3527-33.

25. Wimmer K, Bolliger M, Bago-Horvath Z, Steger G, Kauer-Dorner $D$, Helfgott $R$, et al. Impact of surgical margins in breast cancer after preoperative systemic chemotherapy on local recurrence and survival. Ann Surg Oncol 2020;27:1700-7. [CrossRef]

26. Masuda N, Lee SJ, Ohtani S, Im YH, Lee ES, Yokota I, et al. Adjuvant capecitabine for breast cancer after preoperative chemotherapy. N Engl J Med 2017;376:2147-59. [CrossRef]

27. von Minckwitz G, Huang CS, Mano MS, Loibl S, Mamounas EP, Untch $M$, et al; KATHERINE Investigators. Trastuzumab emtansine for residual invasive HER2-positive breast cancer. N Engl J Med 2019;380:617-28. [CrossRef]

28. Teotia SS, Venutolo C, Haddock NT. Outcomes in patients receiving neoadjuvant chemotherapy undergoing immediate breast reconstruction: effect of timing, postoperative complications, and delay to radiation therapy. Plast Reconstr Surg 2019;144:732e-42e. [CrossRef]

29. Riba J, de Romani SE, Masia J. Neoadjuvant chemotherapy for breast cancer treatment and the evidence-based interaction with immediate autologous and implant-based breast reconstruction. Clin Plast Surg 2018;45:25-31. [CrossRef]

30. Ricci JA, Epstein S, Momoh AO, Lin SJ, Singhal D, Lee BT. A meta-analysis of implant-based breast reconstruction and timing of adjuvant radiation therapy. J Surg Res 2017;218:108-16. [CrossRef]

31. Wormer BA, Valmadrid AC, Kumar NG, Kassis SA, Rankin TM, Higdon KK. Abstract 06: Expediting tissue expansion in implant based breast reconstruction: a comparative study of prepectoral and subpectoral expander placement. Plast Reconstr Surg Glob Open 2018;6:4-5. [CrossRef] 